Mots. Les langages du politique

$71 \mid 2003$

Mondialisation(S)

\title{
Marlène Nasr, Les Arabes et L'Islam vus par les manuels scolaires français
}

Marie Francis-Saad

\section{(2) OpenEdition}

\section{Journals}

Édition électronique

URL : https://journals.openedition.org/mots/8723

DOI : $10.4000 /$ mots. 8723

ISSN : 1960-6001

Éditeur

ENS Éditions

\section{Édition imprimée}

Date de publication : 1 mars 2003

Pagination : 187-192

ISBN : 2-84788-027-5

ISSN : 0243-6450

Référence électronique

Marie Francis-Saad, "Marlène Nasr, Les Arabes et L'Islam vus par les manuels scolaires français ", Mots. Les langages du politique [En ligne], 71 | 2003, mis en ligne le 06 mai 2008, consulté le 23 avril 2022. URL : http://journals.openedition.org/mots/8723 ; DOI : https://doi.org/10.4000/mots.8723 
Marlène NASR, 2001, Les Arabes et L'Islam vus par les manuels scolaires français, Paris, Éditions Khartala, 376 p.

L'étude de M. Nasr a eu pour objet d'extraire et de déterminer les traits caractéristiques de l'image des Arabes et de l'Islam, dans les manuels de lecture, d'histoire, de géographie et d'instruction civique, publiés par quatre éditeurs - Hachette, Nathan, Magnard et Bordas - pour l'enseignement primaire et secondaire en France. La recherche a porté essentiellement sur des manuels utilisés durant l'année 1986, où l'auteure explore les principales dimensions de l'image des Arabes et de l'Islam, dégage deux niveaux d'expression, «en surface» et «profond», relève des stéréotypes, les examine en s'interrogeant: dans quelle mesure la représentation de l'Islam se situe dans le passé ou dans le présent? Dans quelle mesure les manuels examinés établissent une confusion ou une distinction entre les Arabes et l'Islam? Quels sont les grands traits de la civilisation islamique? Les Arabes sont-ils absents ou présents avec leurs mouvements nationaux, dans les textes examinés? L'auteure confrontera Français et Arabes, durant la Conquête islamique, les Croisades, la guerre d'Algérie et les guerres israélo-arabes. Enfin elle observera l'évolution de l'image, dix ans plus tard, selon les variables des manuels.

La première partie du travail porte sur des textes relatifs au monde arabo-islamique, tirés des manuels de lecture du primaire et du secondaire, utilisés en 1986. Parmi les textes du primaire examinés, qui représentent $2 \%$ de l'ensemble et $9 \%$ des textes à thème étranger, trois seulement sont d'origine arabe et maghrébine, l'un tiré des Mille et une nuits et les deux autres d'un roman d'un auteur maghrébin francophone. Malgré les directives du Ministère de l'Éducation, aucun extrait d'auteur arabe contemporain traduit en français ne figure parmi les textes traduits d'une langue étrangère présents dans les manuels (16 \% de l'ensemble). L'image saillante du corpus examiné est celle du désert d'un passé colonial ou encore indéterminé, constate $\mathrm{M}$. Nasr, où les Arabes nomades, identifiés aux Bédouins et aux Maures, se montrent tour à tour «rebelles», « pillards», « saboteurs» (p. 25) et en rapport d'infériorité ou d'hostilité avec les personnages français. Leur image contraste, d'une part, avec celle de leurs interlocuteurs français, brillants, courageux, et d'autre part, avec celle de leurs voisins, les Touaregs, revêtus de qualités glorieuses. Aucune des qualités reconnues aux Bédouins, comme le sens de l'honneur ou celui de l'hospitalité, ne se révèle ici. Les Arabes actuels sont évités, chez eux comme en France. Toutefois des auteurs français, contemporains, dénonceront l'injustice de cette attitude anti-arabe (p. 46). Dans les 
manuels de lecture du secondaire, si la part des textes à thème arabe reste sensiblement la même par rapport à l'ensemble (p. 38), le désert fait place à une diversification de thèmes, dans un cadre spatio-temporel élargi, et l'Arabe reprend une dimension historique, «Sarrazin» au Moyen Age, «Maure» durant la période de la Reconquista, «musulman» ou «Arabe» durant la période coloniale et travailleur immigré aujourd'hui (p. 52). Au terme de cette première partie, M. Nasr estime que l'attraction du désert, des populations déplacées ou misérables, l'absence de la ville arabe, ici, vont de pair avec l'évitement de ce qui rapproche les Arabes actuels, dans leur urbanité, des populations occidentales et apparaissent comme «un refus de la ressemblance» et «une quête» de «la différence» ou de «l'exotique» (p. 60).

La deuxième partie de la recherche aborde les manuels d'histoire du primaire. M. Nasr distingue un discours autocentrique, qui apparait lorsque l'initiative du mouvement est française ou européenne, justifie les Croisades par des motivations religieuses et la colonisation par des intérêts économiques. Mais le ton change pour présenter la Conquête arabe ou la décolonisation, et seul un éditeur, relève M. Nasr, s'efforce, dans un discours «décentré», de faire connaitre les caractères de la religion musulmane et la résistance à la colonisation. Nulle part, on ne trouve le point de vue arabe ou musulman. L'actant arabe est anonyme, enfermé dans une globalité uniforme, dont le nom varie selon les éditeurs et les périodes, «Musulmans », " Arabes », « Sarrazins », « Algériens ». L'appellation «Musulmans» apparait le plus souvent et permet de confondre, sous un même nom, les identifications ethniques, nationales ou linguistiques (p. 97). Dans cette vision monolithique où l'Islam est réduit à sa composante arabe, peu d'auteurs s'attardent sur les non-Arabes parmi les musulmans ou sur les non-musulmans (juifs et chrétiens) parmi les Arabes (p. 147). D'une façon générale, l'Islam se présente, d'une part, à travers une vision positive et amicale, qui souligne les apports considérables de la civilisation islamique et son rôle de pont entre l'Antiquité et le Monde moderne (p. 113), d'autre part, à travers une vision négative qui insiste sur les divisions internes de l'Islam et sa discrimination envers les minorités religieuses (p. 114). Dans ce corpus M. Nasr relève le parti pris, presque général, pour le caractère "esclavagiste» de la société islamique et note qu'aucun auteur ne mentionne que l'esclavage dans l'Islam est en continuité avec les pratiques des civilisations antiques, que personne ne qualifie d'esclavagiste la société américaine, européenne, chrétienne, où le phénomène a duré des siècles (p. 155-156).

L'auteure s'attarde, dans ce contexte historique, sur la guerre d'Algérie 
et distingue, dans son analyse, un clivage entre éditeurs. Les uns voient à l'origine de la guerre la domination et l'exlusion exercées par la société coloniale à l'encontre de la population colonisée, le refus par la minorité européenne d'engager des réformes et d'accorder la citoyenneté aux musulmans et le désir d'indépendance de l'Algérie. Les autres considèrent que l'inégalité démographique, foncière, sociale, culturelle et éducationnelle aurait entrainé l'irruption du nationalisme algérien (p. 175). Tout compte fait, si les manuels soulignent la disproportion numérique entre les victimes du FNL et celles de l'armée française, ils présentent tous la chaine de violence dans le sens d'une initiative de l'acteur algérien, suivie par une riposte de l'acteur français (p. 194).

Du même corpus d'histoire M. Nasr dégage une approche du conflit du Moyen-Orient, centrée sur Israël, qui valorise la société juive émigrée mais ignore les habitants, leur nombre, leur lutte pour l'indépendance de la Palestine en 1919. Les textes ne mettent en avant que leur agressivité vis-à-vis «non pas de la puissance coloniale, mais des colons juifs». Leur mouvement est ainsi transformé en «une attitude anti-juive qui ne peut que provoquer l'antipathie, voire la répulsion» (p. 205). Différente, cette autre approche, développée par un seul manuel, où les auteurs affirment que la responsabilité du «problème palestinien» incombe aux «impérialistes occidentaux» et, par souci d'impartialité, présentent, dans le même dossier, le «Programme du Parti palestinien national arabe» de novembre 1923 et «le mémorandum des Associations islamochrétiennes» de Palestine, en 1919, qui refusent la déclaration Balfour «qui a promis aux sionistes» «une patrie nationale» et «s'opposent à l'immigration juive» dans leur pays (p. 206-207). Sur les guerres israélopalestiniennes, les manuels adhèrent à la vision d'Israël, État «minuscule», menacé par ses voisins, qui veille à «assurer» ses frontières, n'occupe pas, mais "récupère» des territoires, "toujours victorieux», et qui «espère négocier la paix». Les Arabes, belligérants, agresseurs, sont arrogants ou irréalistes car, bien que vaincus, ils «refusent la paix » et s'ils prennent une initiative militaire, ce n'est pas pour libérer leur territoire mais pour «laver les humiliations passées» (p. 217-218). À côté de cette image, quelques remarques, formulées chez un seul éditeur, exposent les deux points de vue, arabe et israélien, dénotant une prise de distance à l'égard du conflit (p. 219).

Dans la troisième partie de l'ouvrage, les manuels de géographie du secondaire soulèvent le problème des immigrés, venus chercher en France des conditions de vie que leurs pays ne peuvent leur offrir, mais les manuels d'éducation civique font apparaitre la cause interne (à la France) 
de l'immigration et précisent que l'État a fait appel à des travailleurs afin de «rester une grande puissance économique après la seconde guerre mondiale» (p. 226-227). Les auteurs incitent les élèves, sous forme de dialogues, de lettres ou de récits, à mieux connaitre leurs camarades étrangers et accepter leurs différences. Peu de manuels insistent sur les ressemblances entre Français et étrangers, tandis que le droit à la différence est scandé avec une telle insistance qu'il est ressenti comme un «éloge de la différence», laquelle n'est, comme dit P.-A. Taguieff, «que la ruse de l'inégalité, le masque de l'intention de discriminer» (p. 230-233). Contrairement aux manuels d'histoire, les manuels de géographie reconnaissent et nomment rarement l'un des manuels, de la grandeur passée des bâtisseurs musulmans, dans le domaine urbain, tout en notant que, si les villes mentionnées dans ce contexte se situent dans la partie arabe du monde musulman, les bâtisseurs ne sont pas identifiés comme arabes et ceci confirme la dichotomie des manuels d'histoire, entre urbanité et arabité (p. 256-257). Dans la géographie des manuels du secondaire, l'intérêt pour l'humain reste abstrait, observe M. Nasr, et se limite aux habitants du désert, qui «envahit» les sujets de l'agriculture, des villes: plusieurs textes portent sur les cultures irriguées dans les oasis ou sur des villes du désert, anciennes et nouvelles. Produit du désert, le pétrole occupe une place de choix dans les textes et l'analyse montre une focalisation sur le marché de ce produit, sur sa consommation, son poids économique dans les pays consommateurs et sur les revenus des pays producteurs. Un éditeur met toutefois en lumière, dans trois manuels, les profits réalisés par les parties moins visibles que les pays producteurs, comme les taxes prélevées par les pays importateurs (p. 244). D’une façon générale, les illustrations, choisies par les manuels, reproduisent largement la répartition thématique des textes, qui privilégie paysages sahariens et activité pétrolière et seule l'attention à la vallée du Nil, à son irrigation, à l'explosion urbaine du Caire, fait exception à la règle (p. 270).

Dans la partie finale de son travail, M. Nasr établit la synthèse des traits de la représentation des Arabes et de l'Islam, parus dans les parties précédentes. Elle constate, dans les manuels de lecture de 1986, que l'espace des Arabes, souvent désertique, est mal délimité et parfois inexistant (p. 285). L'espace urbain est islamique, il n'y a pas de villes arabes, tout comme il n'y a pas d'arts arabes. Les foules urbaines, caractéristiques du monde arabe actuel, sont très peu représentées. M. Nasr revient, dans sa synthèse, sur la question palestinienne, met en relief le changement de vocabulaire où annexion et expansionisme ne sont plus attribués à l'acteur arabe mais à l'acteur israélien, et note que ce point de vue est attribué aux 
Arabes (p. 291). Elle s'attarde de nouveau sur le niveau «profond» des relations entre «les Arabes, acteur négatif» et «les Français, acteur positif», remarquera qu'à l'opposé de cette vision dominante, des critiques, dans le secondaire, dénoncent les actions négatives de l'acteur français, colonisateur (p. 302). Quant aux stéréotypes, comme l'intolérance de l'Islam, le fatalisme et la couardise des Arabes, elle relève qu'ils sont contredits par les auteurs francophones, dont la place réduite dans le corpus atténuera l'effet positif de ces divergences, qui remettent en question la vision dépréciative. Sur la question des immigrés «intrus ou demandés» (p. 307), qui donne lieu à la manifestation la plus actuelle du racisme antiarabe, si des manuels préconisent le retour des immigrés dans leur pays, d'autres proposent l'intégration des immigrés dans la société française (p. 313).

Un dernier chapitre de l'ouvrage porte sur les manuels en cours en 1997-1998. Il explore les changements survenus dans la représentation des Arabes et de l'Islam, extraite des manuels antérieurs (1986), à travers les mêmes matières et cycles et chez les mêmes éditeurs. La lecture au primaire montre que le désert n'est plus le lieu privilégié du récit et que les auteurs francophones mettent en scène des personnages qu'ils identifient par leur nom, leur résidence et leur milieu familial (p. 320). Dans les manuels de lecture du secondaire, l'introduction d'un auteur arabe du $19^{\mathrm{e}}$ siècle, I. Al-Amraoui, représente une innovation et l'Égypte devient «le nouveau lieu de pèlerinage» (p. 325). Dans ce corpus, M. Nasr relève des relations entre Français et Arabes, qui ne sont pas antagonistes et la vision de l'Islam apparait, dans ces manuels actuels, moins divergente, plus positive, puisée dans des extraits du Coran ou d'auteurs arabes, tels que AlTabari, Ibn Jubayr, Avicenne. Si certains de ces manuels donnent la priorité à l'emprunt par les savants musulmans de l'héritage antique et sa «transmission» à l'Occident, les autres developpent davantage la part des inventions et apports de la civilisation islamique et presque tous n'oublient pas d'insister sur sa diversité ethnique (p. 329-331). Dans ce réajustement, la société islamique n'est plus considérée comme «une société esclavagiste». Des changements apparaissent également dans le discours sur la guerre d'Algérie. L'enchainement causal univoque, qui présentait l'action française comme une riposte à une initiative algérienne, est rompu et l'interaction entre les positions politiques est analysée (p. 339). Dans l'histoire contemporaine du Moyen-Orient, les manuels actuels continuent à fonder le nationalisme arabe sur l'appartenance de la majorité des Arabes à la religion musulmane, plutôt qu'à leur partage d'une langue commune. «Le réveil islamique» occupe une plus grande place 
dans les manuels actuels, critiques vis-à-vis de "l'intégrisme islamique» (p. 342). Par rapport au conflit du Moyen-Orient, les textes actuels semblent avoir pris de la distance et le stéréotype, Israël toujours vainqueur/les Arabes toujours vaincus, déjà remis en question par une vision minoritaire, n'est plus de mise. Les auteurs reconnaissent que «l'offensive» arabe de 1973 a remporté un franc succès et que, pour la première fois, une armée arabe «a vaincu même provisoirement Israël». Un autre cliché reste tenace, celui de l'humiliation et de la revanche qui motiveraient les guerres de 1948 et 1973, dont l'objectif politique de libération n'est pas évoqué. Parler de libération, c'est admettre l'illégitimité de l'occupation (p. 348). Somme toute, l'espace arabe n'a plus seulement les traits du nomadisme, du désert, du pétrole et des migrations; il se concentre plutôt sur le monde urbain, les villes arabes peuplées, la production et entérine l'association de l'arabité à l'urbanité. Un changement apparait ainsi par rapport au corpus antérieur et constitue un réel progrès sur la voie d'un réexamen de l'image des Arabes et de l'Islam.

À travers des manuels de 1986 et un correctif apporté plus tard, l'analyse de M. Nasr a dégagé une vision plurielle de l'Islam, des Arabes, des confrontations franco-arabes, de la guerre d'Algérie, du conflit israélopalestinien, de l'immigration, puis a examiné les permanences et les changements de cette image. Son approche a souligné notamment les points de similitude entre les éditeurs ou leurs divergences. Le destinataire du travail y gagne une capacité à mieux saisir la spécificité des relations de deux collectivités humaines historiques, tout comme il se construit une revision des Arabes et de l'Islam. En fait, cet ouvrage bien documenté, suscite la réflexion, même si l'on peut ne pas être d'accord sur tel ou tel point. Soucieuse d'objectivité, de lucidité, M. Nasr a mené son étude avec maitrise, constamment précise dans ses enquêtes et ses analyses de discours. Cet ouvrage a toutes les chances de rester une contribution majeure à un dialogue entre les civilisations, où chacun pourra sentir la nécessité d'accepter l'Autre, dans sa différence comme dans sa similitude, et de mettre, dans la mesure de ses moyens, un bémol à l'agitation de l'actualité.

Marie Francis-Saad 